同位素稀释-气相色谱-串联质谱法测定土壤中的指示性毒杀芬

\author{
张 兵, 吴嘉嘉, 刘国瑞, 高丽荣, 郑明辉* \\ (中国科学院生态环境研究中心, 北京 100085)
}

摘要 :建立了土壤样品中指示性毒杀芬 Parlar No. 26 (P26)、Parlar No. 50 ( P50) 和 Parlar No. 62 (P62)的同位素 稀释-气相色谱-串联质谱 (ID-GC-MS/MS) 的分析方法。土壤样品使用压力溶剂萃取装置 (PLE) 提取, 以丙酮-正 己烷 $(1: 1, \mathrm{v} / \mathrm{v})$ 混合溶液为提取溶剂; 提取液依次经由多层酸性硅胶柱和活化硅胶柱净化;洗脱液经氮吹浓缩至 $20 \mu \mathrm{L}$ 后, 利用 GC-MS/MS 的多反应监测 (MRM) 模式进行定性和定量。结果表明本方法可对样品中的 P26、P50 和 P62 进行分析, 相对标准偏差 (RSD) 小于 $11 \%$, 回收率可以达到 55\% 110\%;P26、P50 和 P62 的仪器检出限分别 为 3.0 、 3.0 和 $6.0 \mathrm{pg}$ 。将该方法用于某地区农田表层土壤中 3 种指示性毒杀芬的检测, 其中 P26 的含量为 0.17 $n g / g 、 P 50$ 为 $0.08 \mathrm{ng} / \mathrm{g} 、 \mathrm{P} 62$ 为 $0.09 \mathrm{ng} / \mathrm{g}$ 。此方法适用于土壤样品中指示性毒杀芬的分析。

关键词 :同位素稀释法;气相色谱;串联质谱;指示性毒杀芬;土壤

中图分类号:O658 文献标识码 :A 文章编号 :1000-8713(2010)05-0456-04

\title{
Determination of indicator toxaphene in soil by isotope dilution-gas chromatography-tandem mass spectrometry
}

\author{
ZHANG Bing , WU Jiajia , LIU Guorui , GAO Lirong , ZHENG Minghui * \\ (Research Center for Eco-Environmental Sciences ,Chinese Academy of Sciences , Beijing 100085 ,China)
}

\begin{abstract}
Although toxaphene is now banned in use , the analysis of toxaphene has attracted increasing interest due to its persistence and widespread atmospheric transport in the environment. A new method based on isotope dilution-gas chromatography-tandem mass spectrometry ( ID-GC-MS/MS) has been developed for the determination of three toxaphene specific congeners comprised of Parlar No. 26 ( P26) , Parlar No. 50 ( P50) and Parlar No. 62 ( P62) in soil. ${ }^{13} \mathrm{C}_{10}$-labeled indicator toxaphene solution was added to the sample prior to pretreatment. Then the sample was extracted using pressurized liquid extraction ( PLE) followed by purification on multilayer acidic silica column and neutral silica column. The eluent was concentrated under gentle nitrogen gas flow and spiked with the injection of internal standard of ${ }^{13} \mathrm{C}_{10}$-chlordane. Identification and quantification of the analytes were carried out in the multiple reaction monitoring ( MRM) mode after the GC separation. The linear range was $20-800 \mu \mathrm{g} / \mathrm{L}$ for three congeners, limits of detection (LOD) ranged from 3.0 to 6.0 pg. The five point calibration curves showed a good linearity for all the congeners $\left(R^{2}>0.99\right)$. The relative standard deviations ( RSDs) were below $11 \%$ for and the spiked recoveries were in the range of $55 \%-110 \%$. The developed analytical method is suitable for the determination of toxaphene specific congeners in soil.
\end{abstract}

Key words : isotope dilution ; gas chromatography ( GC) ; tandem mass spectrometry ( MS/ MS) ; indicator toxaphene ; soil

毒杀芬是一种广谱性杀虫剂,由 $5 \sim 10$ 个氯取 代的葫烯或茨烷组成，根据其结构式理论推断，它的 同类物的数量多达 32768 种 $^{[1]}$ 。在 20 世纪 70 年
代，毒杀芬被用作滴滴涕( DDT) 的替代品, 为了保 护农作物、家禽和家畜免受害虫和病毒侵害,曾经在 世界范围内被广泛使用 ${ }^{[2]}$ 。由于它具有较高的毒 
性、持久性和生物富集性,所以可在大气中进行远距 离传输 ${ }^{[3]}$, 在远离使用地点的海洋哺乳动物体内、 鱼类甚至是人乳中都检测到了不同浓度的毒杀 芬 ${ }^{[4-6]}$;毒杀芬已被列为《斯德哥尔摩公约》中首批 控制的 12 种持久性有机污染物 (persistent organic pollutants，POPs）之一。虽然毒杀芬同类物数量 众多, 但有研究 ${ }^{[7]}$ 证明只有七氯 九氯的同系物会 在脂肪中高富集，据文献报道，动物脂肪中的 Parlar No. 26 ( P26) 、 Parlar No. 50 ( P50) 和 Parlar No. 62 (P62) 3 种毒杀芬 ${ }^{[8]}$ 的含量大约占总量的 $8 \%$ $50 \%$,因此通常将这 3 种毒杀芬同类物作为指示性 毒杀芬加以研究。毒杀芬与其他 POPs 性质相似, 它的 $\log K_{\mathrm{oc}}$ 为 $2.5 \sim 6$,会被土壤颗粒物吸附, 土壤 中的毒杀芬还可能污染地下水 ${ }^{[9]}$,所以毒杀芬被认 为是最受关注的有机氯农药 ${ }^{[10]}$ 。

环境中的毒杀芬残留量较低,因此需要高选择 性、高灵敏度的检测方法。由于电子捕获检测器 ( ECD) 对于含氯的有机物具有较高的灵敏度，因此 安装 ECD 的气相色谱仪曾被广泛应用于环境介质 中残留毒杀芬的定性和定量分析 ${ }^{[11,12]}$ 。但由于 ECD 对目标化合物选择性不强, 近年来在环境样品 中毒杀芬的检测多采用气相色谱-质谱联用 (GCMS) 方法。de Boer 等 ${ }^{[13]}$ 利用负化学电离源 GCMS 技术在鱼和海洋哺乳动物等生物样品中检测到 了不同浓度水平的毒杀芬。由于毒杀芬同类物数量 众多,并且基质或其他相似物质可能会对目标化合 物的测定产生严重干扰，易产生假阳性结果，因此需 要更准确的分析方法。同位素稀释法 (ID) 被认为 是环境介质中 POPs 定性和定量最准确的方法 ${ }^{[14]}$, 目前在二恶英类和多氯联苯的分析方法中已得到广 泛应用。2009 年 8 月美国 CIL 公司推出 ${ }^{13} \mathrm{C}_{10}$ 标记 P26、P50 和 P62 指示性毒杀芬标准品, 为采用 ID 法进行毒杀芬检测提供了可能。基于 GC-MS/MS 技术检测毒杀芬的最新进展 ${ }^{[8]}$, 我们建立了 ID-GCMS/MS 法检测土壤中指示性毒杀芬的分析方法。 采用压力溶剂萃取 (PLE) 技术 ${ }^{[15]}$ 对样品中的毒杀 芬进行提取，利用多层酸性硅胶柱对样品进行净化， 用活化硅胶柱消除样品中多氯联苯和其他有机氯农 药的干扰，并对 3 种指示性毒杀芬同类物分析过程 中的各项指标, 如回收率、空白、基质加标和平行样 等质量保证和质量控制的措施进行研究。

\section{1 实验部分}

\section{1 仪器、试剂与材料}

7000A 型三重四极杆串联质谱仪 (美国 Agilent
公司);压力溶剂提取仪（美国 FMS 公司）;氮吹浓 缩仪（美国 Organomation Associates Inc.）;旋转 蒸发仪 (瑞士 BüCHI 公司) ;冷冻干燥机 ( 北京松源 华兴科技公司)。

${ }^{13} \mathrm{C}_{10}$-氯丹、 ${ }^{13} \mathrm{C}_{10}$ 标记和非标记的 P26、P50 和 P62 标准物质 (纯度 $99 \%$,美国 CIL 公司 ) ; 实验所用 溶剂甲醇、丙酮、二氯甲烷、正己烷及甲苯均为农残 级 (美国 J. T. Baker 公司) 硫酸 ( 优级纯, 北京北化 精细化学品公司)。硅胶(100 200 目，山东青岛海 洋化工厂分厂) 经甲醇和二氯甲烷柱淋洗后氮气吹 干。硅胶使用前 $12 \mathrm{~h}$ 于 $180{ }^{\circ} \mathrm{C}$ 活化 $6 \mathrm{~h}$ 后, 置于干 燥器中待用;22\% 酸性硅胶:取活化后的硅胶 $78 \mathrm{~g}$ 逐滴加入浓硫酸 $22 \mathrm{~g}$,边加边振荡,使其分散均匀。 将制备好的酸性硅胶放入试剂瓶中密封，保存在干 燥器中; $44 \%$ 酸性硅胶:取活化后的硅胶 $78 \mathrm{~g}$, 逐滴 加入浓硫酸 $44 \mathrm{~g}$, 边加边振荡，使其分散均匀。将制 备好的酸性硅胶放入试剂瓶中密封, 保存在干燥器 中;无水硫酸钠: 优级纯, 使用前于马弗炉中 $660{ }^{\circ} \mathrm{C}$ 灼烧 $8 \mathrm{~h}$ 。

\section{2 样品前处理}

\subsection{1 压力溶剂提取}

将土壤样品冷冻干燥后, 研磨, 取 $20.0 \mathrm{~g}$ 加入 $10.0 \mu \mathrm{g} / \mathrm{L}$ 的 ${ }^{13} \mathrm{C}_{10}$ 标记 P26、P50 和 P62 标准品的丙 酮溶液, 并与适量硅藻土混匀后转移至 $100 \mathrm{~mL}$ 提 取筒中，将提取筒静置 $30 \mathrm{~min}$ 后放入 PLE 提取筒 的加热套内，以丙酮-正己烷 $(1: 1, \mathrm{v} / \mathrm{v})$ 混合溶液为 提取溶剂。提取温度 : 100 ${ }^{\circ} \mathrm{C}$;系统压力 :15 $\mathrm{MPa}$;加 热时间: 15 min ;氮吹时间 $30 \mathrm{~s}$ 。循环 2 次。

\subsection{2 多层酸性硅胶柱净化}

将 PLE 提取液在旋转蒸发仪上浓缩至约 2 $\mathrm{mL}$, 并转移至多层酸性硅胶柱上 $(200 \mathrm{~mm} \times 10 \mathrm{~mm}$, 干法装柱，从上到下依次为 $5.0 \mathrm{~g}$ 无水硫酸钠、 $2.5 \mathrm{~g}$ 活化硅胶、10.0 g 22\% 酸性硅胶和 $12.5 \mathrm{~g} 44 \%$ 酸性 硅胶)，用 $100 \mathrm{~mL}$ 正己烷淋洗多层酸性柱，收集洗 脱液, 浓缩至 $2 \mathrm{~mL}$,准备过活化硅胶柱。

\subsection{3 活化硅胶柱净化}

将多层酸性硅胶柱淋洗浓缩液转移至活化硅胶 柱上 $(200 \mathrm{~mm} \times 10 \mathrm{~mm}$,干法装柱，从上到下依次为 $5.0 \mathrm{~g}$ 无水 $\mathrm{Na}_{2} \mathrm{SO}_{4}$ 和 $8.0 \mathrm{~g}$ 活化硅胶), 用 $45 \mathrm{~mL}$ 正己烷淋洗, 去除多氯联苯及其他有机氯农药的干 扰，再用 $50 \mathrm{~mL}$ 甲苯-正己烷 $(35: 65, \mathrm{v} / \mathrm{v})$ 溶液淋 洗, 收集洗脱液, 氮吹浓缩至约 $20 \mu \mathrm{L}$, 然后加入 10 $n g$ 的 ${ }^{13} \mathrm{C}_{10}$-氯丹作为进样内标。

\section{3 色谱条件}

采用 DB-5MS 色谱柱 $(30 \mathrm{~m} \times 0.25 \mathrm{~mm} \times 0.25$ 
$\mu \mathrm{m})$,载气氦气, 恒流模式, 流速 $1 \mathrm{~mL} / \mathrm{min}$;脉冲 不分流进样 $1 \mu \mathrm{L}$ 。进样口温度 $: 230{ }^{\circ} \mathrm{C}$; 传输线温 度:290 ${ }^{\circ} \mathrm{C}$; 程序升温: $80{ }^{\circ} \mathrm{C}$ 保持 $2 \mathrm{~min}$, 以 20 ${ }^{\circ} \mathrm{C} / \mathrm{min}$ 速率升至 $200{ }^{\circ} \mathrm{C} 5{ }^{\circ} \mathrm{C} / \mathrm{min}$ 升至 $250{ }^{\circ} \mathrm{C}$ 保持 $7 \mathrm{~min}$, 再以 $10{ }^{\circ} \mathrm{C} / \mathrm{min}$ 的速率升至 $300{ }^{\circ} \mathrm{C}$ 。

\section{4 质谱条件}

电子轰击源 $(\mathrm{EI})$, 电子能量 $50 \mathrm{eV}$, 源温度 230 ${ }^{\circ} \mathrm{C}$, 双四极杆温度 : $150{ }^{\circ} \mathrm{C}$, 碰撞气 : He $2.25 \mathrm{~mL} / \mathrm{min}$ 、 $\mathrm{N}_{2} 1.5 \mathrm{~mL} / \mathrm{min}$ 。采用多反应监测 (MRM) 方式进行 数据采集,毒杀芬同类物的监测离子 $(m / z)$ 及碰撞 电压 (CE) 如表 1 所示。

表 1 MRM 模式下指示性毒杀芬的 监测离子及碰撞电压 $(\mathbf{C E})$

Table 1 Compounds monitored mass and collision energy ( CE), using electronic ionization

\begin{tabular}{|c|c|c|c|c|}
\hline \multirow[b]{2}{*}{ Compound } & \multirow{2}{*}{$\begin{array}{c}\text { Precursor } \\
\text { ion } \\
(m / z)\end{array}$} & \multicolumn{2}{|c|}{ Product ion $(\mathrm{m} / \boldsymbol{z})$} & \multirow{2}{*}{$\begin{array}{c}\mathrm{CE} / \\
\mathrm{eV}\end{array}$} \\
\hline & & $\begin{array}{c}\text { Quantitative } \\
\text { ion }\end{array}$ & $\begin{array}{l}\text { Qualitative } \\
\text { ion }\end{array}$ & \\
\hline $\mathrm{P} 26$ & 195 & 195.1 & 159.1 & 5 \\
\hline P50 & 195 & 195.1 & 159.1 & 5 \\
\hline P62 & 195 & 195.1 & 159.1 & 5 \\
\hline${ }^{13} \mathrm{C}_{10}-\mathrm{P} 26$ & 166 & 166.1 & 130.1 & 15 \\
\hline${ }^{13} \mathrm{C}_{10}-\mathrm{P} 50$ & 166 & 166.1 & 130.1 & 15 \\
\hline${ }^{13} \mathrm{C}_{10}-\mathrm{P} 62$ & 166 & 166.1 & 130.1 & 15 \\
\hline${ }^{13} \mathrm{C}_{10}$-Chlordane & 385 & 276.0 & 242.0 & 30 \\
\hline
\end{tabular}

\section{5 质量保证与质量控制}

PLE 提取筒和玻璃器血使用之前分别用丙酮、 甲醇和二氯甲烷超声清洗干净, 去除背景有机杂质, 每个样品中均加 ${ }^{13} \mathrm{C}_{10}$ 同位素标记的 P26、P50、 P62 和氯丹, 以评价分析方法的回收率。

\section{2 结果与讨论}

\section{1 校正曲线}

根据文献报道的环境背景值样品中毒杀芬的含 量范围，本实验确定并配制了 3 种指示性毒杀芬的 标准溶液, 其中同位素标记内标物 ${ }^{13} \mathrm{C}_{10}-\mathrm{P} 26 、{ }^{13} \mathrm{C}_{10}$ $\mathrm{P} 50$ 及 ${ }^{13} \mathrm{C}_{10}-\mathrm{P} 62$ 的质量浓度为 $200 \mu \mathrm{g} / \mathrm{L}$, 进样内标 ${ }^{13} \mathrm{C}_{10}$ 标记的氯丹的质量浓度为 $200 \mu \mathrm{g} / \mathrm{L}$,工作曲线 中 P26、P50 及 P62 的质量浓度范围为 $20 \sim 800$ $\mu \mathrm{g} / \mathrm{L}$ 。经计算，各标记化合物和非标记化合物之间 的相对响应值 (RR) 的相对标准偏差 (RSD) 在 4\% $\sim 15 \%$ 之间 5 点校正曲线具有良好的线性。

\section{2 精密度和回收率}

称取 3 份 $20.0 \mathrm{~g}$ 石英砂与适量的硅藻土均匀 混合, 分别加入质量浓度为 $400 \mu \mathrm{g} / \mathrm{L}$ 的 P26、P50 及 $\mathrm{P} 62$ 的标准溶液和质量浓度为 $200 \mu \mathrm{g} / \mathrm{L}$ 的 ${ }^{13} \mathrm{C}_{10}$ $\mathrm{P} 26 、{ }^{13} \mathrm{C}_{10}-\mathrm{P} 50$ 及 ${ }^{13} \mathrm{C}_{10}-\mathrm{P} 62$ 内标物, 按 1.2 节所述的
前处理方法, 对加标的基质样品进行提取净化。分 析结果表明 ,P26、P50 和 P62 的质量浓度依次为 $(400$ $\pm 6.2) 、(400 \pm 10.3)$ 和 $(400 \pm 20.4) \mu \mathrm{g} / \mathrm{L}$;同位素标 记物的回收率为 $55 \% \sim 110 \% ; \mathrm{RSD}$ 为 $3.2 \% \sim 10.1 \%$ 。

2.3 空白试验

空白试验结果表明，样品提取时加入 ${ }^{13} \mathrm{C}_{10}-\mathrm{P} 26 、{ }^{13} \mathrm{C}_{10}-\mathrm{P} 50$ 和 ${ }^{13} \mathrm{C}_{10}-\mathrm{P} 62$ 的同位素稀释剂溶 液, 回收率为 $65 \% \sim 115 \%$, 空白样品中 P $26 、$ P50 及 P62 的含量低于方法检出限, 不影响实际样品的 定量。

\section{4 方法检出限}

将标准溶液稀释,进样 $1 \mu \mathrm{L}$ 测试, 以毒杀芬同 类物的信噪比 $(S / N)$ 约为 3 平行测定 6 次,计算 P26、P50 和 P62 的绝对检出限分别为 3.0、3.0 和 $6.0 \mathrm{pg}$ 。

\section{5 实际土壤样品的测定}

采用 ID 法对某地区农田表层土壤中的指示性 毒杀芬进行了分析，实验结果(见图 1 ) 表明，同位素 内标 ${ }^{13} \mathrm{C}_{10}-\mathrm{P} 26 、{ }^{13} \mathrm{C}_{10}-\mathrm{P} 50$ 及 ${ }^{13} \mathrm{C}_{10}-\mathrm{P} 62$ 的回收率为 $50 \%$ 110\%。该土壤样品中指示性毒杀芬 P26、P50 和 P60 的含量分别为 $0.17 、 0.08$ 和 $0.09 \mathrm{ng} / \mathrm{g}$ 。
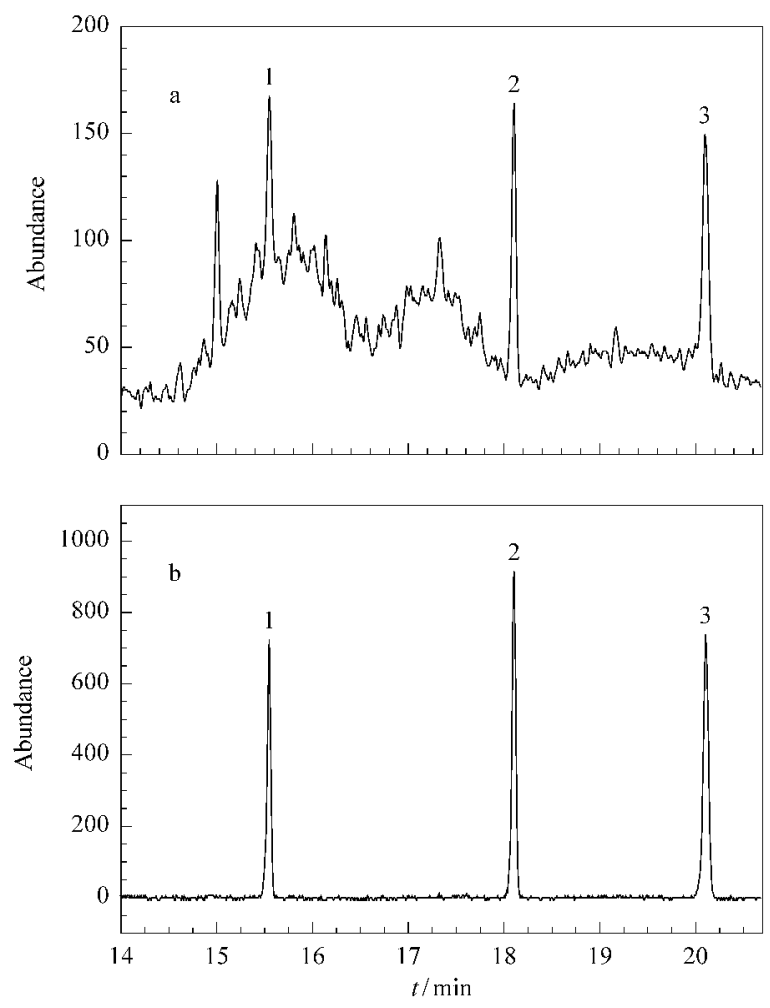

图 1 (a) 实际土壤样品和 $(\mathbf{b})$ 标准溶液中 指示性毒杀芬的色谱图

Fig. 1 Chromatograms of P26, P50 and P62 in (a) a soil sample and $(b)$ standard solution

a. 1. $\mathrm{P} 26$;2. $\mathrm{P} 50$; 3. P62. b. $1 .{ }^{13} \mathrm{C}_{10}-\mathrm{P} 26 ; 2 .{ }^{13} \mathrm{C}_{10}-\mathrm{P} 50$; 3. ${ }^{13} \mathrm{C}_{10}-\mathrm{P} 62$ 


\section{3 结语}

本研究建立了 ID-GC-MS/MS 检测指示性毒杀 芬的分析方法。该方法灵敏，精密度高。对土壤样 品的分析验证表明，该方法适用于环境土壤样品中 的指示性毒杀芬 P26、P50 和 P62 的分析。

\section{参考文献：}

[1] Vetter W. Chemosphere , 1993, 26:1079

[2] Saleh M A. Rev Environ Contam Toxicol , $1991,118: 1$

[3] Paasivirta J, Sinkkonen S, Nikiforov V, et al. Environ Sci Pollut Res , 2009 , $16: 191$

[4] Andersson Ö , Linder C E , Olsson M , et al. Arch Environ Contam Toxicol , $1988,17: 755$

[ 5 ] Alder L , Beck H , Khandker S , et al. Chemosphere , 1997 , $34: 1389$

[6] Gouteux B , Lebeuf M , Hammill M O, et al. Environ Sci Technol , $2005,39: 1448$
[7] Alder L, Vieth B. Fresenius J Anal Chem , 1996 , 354 : 81

[8] Veyrand B , Venisseau A , Marchand P, et al. J Chromatogr B , 2008, $865: 121$

[9] Bezchlebová J , Černohlávková J , Lána J , et al. Ecotoxicol Environ Saf , $2007,68: 326$

[10] IFCS , Intergovernmental Forum on Chemical Safety. Final Report of the IFCS Ad Hoc Working Group on Persistent Organic Pollutants. [2009-12-19]. http ://www. chem. unep. ch/pops/indxhtms/manwgrp. html

[11] Liu J S , Liu H Y , Zhang H, et al. Environmental Science and Technology (刘婕丝, 刘红玉, 张慧, 等. 环境科学与技 术), 2007, 30(10):90

[12] Wang M T , Liu Z Y , Mu J , et al. Dyeing \& Finishing (王明 泰，刘志研，牟峻，等. 印染), 2006,6:37

[13] de Boer J , Wester P G. Chemosphere ,1993,27(10) :1879

[14] Jones K C , de Voogt P. Environ Pollut , 1999 , 100 : 209

[15 ] Xie Y L , Rao Z , Wang X H , et al. Journal of Instrumental Analysis (谢原利, 饶竹, 王晓华, 等. 分析测试学报), $2009,28(7): 804$ 